\title{
Differential neuronal responses to the self and others in the extrastriate body area and the fusiform body area
}

\author{
SILJA Vocks \\ Ruhr University Bochum, Bochum, Germany \\ Martin BusCh ANd Dietrich GRÖNEMEYeR \\ University of Witten/Herdecke, Witten, Germany \\ AND \\ Dietmar Schulte, Stephan Herpertz, and Boris Suchan \\ Ruhr University Bochum, Bochum, Germany
}

\begin{abstract}
The extrastriate body area (EBA) and the fusiform body area (FBA) are selectively activated by viewing human bodies. However, the role of these functionally defined brain areas in self-other discrimination is still unresolved. Thirty-one females were presented with 16 pictures of their own body and another body in a bikini and with scrambled images while fMRI was performed. Moreover, standardized stimulus material was used in order to localize the EBA and FBA. Region-of-interest analysis showed an enhanced BOLD response to the self-images relative to the non-self-images in the right EBA and FBA. The right EBA and FBA are activated differently by viewing oneself and others and might thus play a role in visual self-other discrimination.
\end{abstract}

Since humans are a highly social species, we devote a great deal of attention to others surrounding us, meaning that self-other discrimination is crucial for social functioning. Consequently, the neuronal basis of general selfreferential processing in various domains has been extensively studied in cognitive neuroscience (for a review, see Northoff et al., 2006). However, specific knowledge about the neural systems underlying visual self-other discrimination is limited. Evidence from recent research suggests that multiple brain areas seem to be involved in self-recognition (Sugiura et al., 2006). For example, a recent meta-analysis integrating the findings of nine fMRI studies indicated that the left fusiform gyrus, the bilateral middle and inferior frontal gyri, and the right precuneus were consistently activated by visual processing of one's own face (Platek, Wathne, Tierney, \& Thomson, 2008). In contrast to the relatively high number of studies on visual self-perception of the face, the processing of the rest of the body has not received as much attention in cognitive neuroscience, even though the body also provides substantial information, including identity, gender, and emotion (Chan, Peelen, \& Downing, 2004; Gillihan \& Farah, 2005).

In the context of the visual processing of the human body in general, distinct regions in the human cortex that respond selectively to certain body stimuli have been identified. Whereas the fusiform face area (FFA) and the occipital face area (OFA) respond to human faces (Dri- cot, Sorger, Schiltz, Goebel, \& Rossion, 2008; Kanwisher, McDermott, \& Chun, 1997; Puce, Allison, Asgari, Gore, \& McCarthy, 1996), the extrastriate body area (EBA) and the fusiform body area (FBA) are activated by viewing images of the human body (Downing, Jiang, Shuman, \& Kanwisher, 2001; Peelen \& Downing, 2007). The EBA is bilaterally located in the lateral occipitotemporal cortex, and the FBA can be found adjacent to the FFA in the fusiform gyrus (Downing, Chan, Peelen, Dodds, \& Kanwisher, 2006; Peelen \& Downing, 2007). These two body-selective brain areas respond to a wide range of visual body depictions. For example, it was demonstrated that EBA activity is elicited by visual stimulation with images of humans presented in various formats, such as photographs, stick figures, or silhouettes. Moreover, the EBA response to nonhuman mammals has been shown to be weaker than that to human bodies, but stronger than that to objects (e.g., Downing et al., 2001), suggesting that the EBA activation is elicited by objects that have a body plan resembling that of human beings (Peelen \& Downing, 2007).

The selectivity of the EBA and of the FBA for the human figure raises the question of whether these two body-selective brain areas have properties of extracting visual information about oneself and other individuals and thus play a role in self-other discrimination. For the EBA, identity-dependent differences in the processing of one's 
own body and other complete bodies have been tested in three studies to date (Chan et al., 2004; Hodzic, Muckli, Singer, \& Stirn, 2009; Saxe, Jamal, \& Powell, 2006). Two of them differentiated between an egocentric viewpoint, which is consistent with the usual perspective when one looks at one's own body, and an allocentric viewpoint, which is in accordance with the perspective of looking at another person. In both studies, an enhanced activation of the right EBA was found from the allocentric perspective when bodies (Chan et al., 2004) or hands and feet (Saxe et al., 2006) were viewed, whereas in the EBA of the left hemisphere, no such viewpoint-dependent difference was observed. In contrast to Saxe et al., who did not use photographs of the participants themselves, Chan et al. further differentiated between pictures of oneself and those of another person and did not find differences in the EBA activation in either hemisphere. This result is in line with the findings of a recent study by Hodzic et al., in which the comparison of neuronal responses to looking at one's own body and another's body did not reveal different activations in the EBA. From these results, it was concluded that the EBA is involved in the analysis of body-related information in general but that the properties selected by the EBA might not be sufficiently detailed to differentiate between self and others and, thus, the EBA does not seem to have an ability to recognize oneself (Chan et al., 2004; Hodzic et al., 2009). However, in another study in which exclusively body parts and no complete bodies were used as stimulus materials (Myers \& Sowden, 2008), a more pronounced response in the right EBA was found, indicating that the EBA might be selectively sensitive to images of one's own body and another person's body. For this reason, it has to be considered that in the studies conducted so far in which complete bodies were used as stimulus materials, relatively small and heterogeneous samples of participants of both sexes were examined and the statistical approaches used might not have been sufficiently sensitive to detect possibly existing moderate stimulusspecific differences.

On the basis of these considerations, the aim of the present study was to investigate, with female participants, differences in the processing of photographs of one's own body and another female's body with a larger and more homogeneous sample. In addition, positive and negative affect elicited by looking at the various body stimuli was considered, since self-relatedness has been shown to be associated with the valence and intensity of emotions in subcortical brain regions underlying emotion processing (Northoff et al., 2009). Taking into account previous research (Myers \& Sowden, 2008), we hypothesized that in a region-of-interest (ROI)-based analysis, looking at one's own body would lead to a more pronounced activity in the EBA and FBA, as compared with viewing another female. Furthermore, on the basis of the results of a meta-analysis integrating the findings of studies on visual self-recognition regarding one's face (Platek et al., 2008), we predicted that in a whole-brain analysis, viewing oneself would elicit a stronger reaction in the precuneus and the middle and inferior frontal gyrus, as compared with looking at another female's body.

\section{METHOD}

\section{Sample}

The sample consisted of $N=31$ healthy females, who were recruited by university announcements. Inclusion criteria for participation in the study were female sex, an age of 18-50 years, and being right-handed, as assessed by the Edinburgh Inventory (Oldfield, 1971). Persons were excluded if they had metal parts in their body, were pregnant, had a history of neurological disease, or suffered from claustrophobia. Furthermore, females with obesity or any form of eating disorder were excluded from the study on the basis of their scores on the Eating Disorder Examination Questionnaire (Fairburn \& Cooper, 1993; Hilbert, Tuschen-Caffier, Karwautz, Niederhofer, $\&$ Munsch, 2007), since previous research had demonstrated abnormalities in the EBA morphology (Suchan et al., 2010) and functionality (Uher et al., 2005) of individuals with eating disorders.

\section{Stimuli}

For the present study, one set of photographs of the participant's own body and one set of photographs of the body of an unknown female were used. In addition, one set of scrambled images (puzzle) was included, which was generated from the pictures of the unknown female's body in order to provide a visual input comparable to the target stimuli. For each participant, the photographs were taken in the same room under standardized conditions, with the participants wearing a uniform pink bikini of the same style from the same manufacturer in their size. Each participant was photographed from 16 perspectives (front, back, right, left, and the 12 between perspectives) without the head, since previous research had demonstrated that exposing the face decreased activation of the EBA (Morris, Pelphrey, \& McCarthy, 2006). The photographs of the participant's own body and those of the other female's body, as well as the scrambled images, were presented using a block design without repetition of the same block. Each of the three sets of 16 photographs was presented three times. Within the blocks, each photograph was presented for $3 \mathrm{sec}$ in a random order, resulting in a duration of $48 \mathrm{sec}$ for one block. Before each block, a slide was shown that indicated which kind of pictures would be shown next (see Figure 1). Between each block, a fixation cross was presented for $48 \mathrm{sec}$ in order to enable the BOLD response to return to the baseline. In addition, after the above-mentioned sets of stimuli had been presented, standardized pictures of bodies (photographed without the head) and chairs, as provided by Downing et al. (2001), were presented to the participants in order to locate the EBA (www.bangor.ac.uk/ pss811/ page $7 /$ page $7 . \mathrm{html}$ )

\section{Image Acquisition}

Images were acquired on a 1.5-T Symphony scanner (Siemens, Erlangen, Germany). A total of 440 T2-weighted EPI images covering the whole brain and omitting parts of the cerebellum were acquired in each condition. Each volume consisted of 25 slices of $3 \mathrm{~mm}$ (interslice gap: $1 \mathrm{~mm}$ ). The repetition time (TR) was $80 \mathrm{msec}$ per slice (total TR $=2,000 \mathrm{msec}$ ), with an echo time (TE) of $40 \mathrm{msec}$ and a flip angle of $90^{\circ}$. In addition, high-resolution T1-weighted images with a voxel size of $1 \times 1 \times 1 \mathrm{~mm}$, using a TR $=2,110 \mathrm{msec}$, a TE $=3.93 \mathrm{msec}$, and a flip angle of $15^{\circ}$, were acquired to support localization and coregistration of the functional data.

\section{Questionnaire Data}

For the purpose of assessing positive and negative affect when the participants looked at photographs of their own body and the other body presented on a computer screen directly after the scanning session, the Positive and Negative Affect Schedule (Krohne, Egloff, Kohlmann, \& Tausch, 1996; Watson, Clark, \& Tellegen, 1988) 


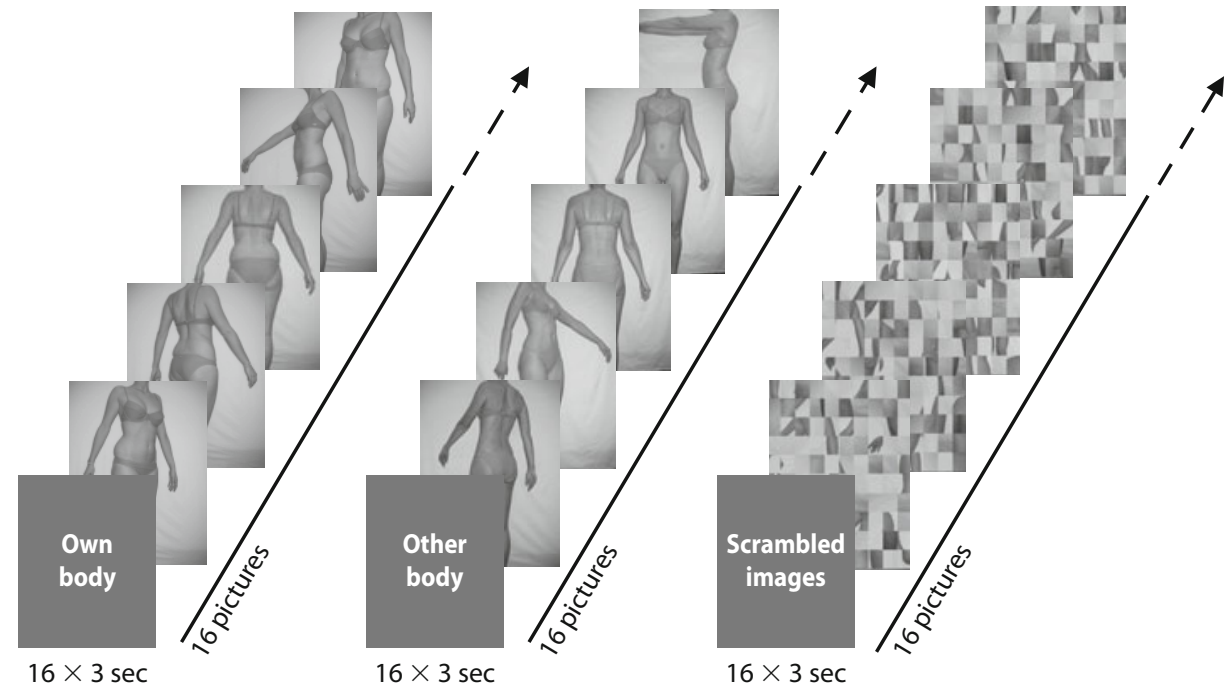

Figure 1. Stimulus materials.

was administered. The scale is composed of 20 items, of which 10 items cover positive affect (e.g., "interested") and 10 items refer to negative affect (e.g., "scared"). The participants had to indicate, on a scale ranging from 1 (not at all) to 5 (very much), the extent to which each of the listed thoughts emerged during the presentation of the pictures. The internal consistency on the Positive and Negative Affect Schedule (Cronbach's $\alpha$ ) ranges from $\alpha=.85$ to $\alpha=.86$.

\section{Procedure}

The participants were informed about the study and gave their written consent. Afterward, each participant was photographed from 16 perspectives under strictly constant conditions. Following this, the scanning session was performed, and each participant was asked whether she had looked at the pictures and had not closed her eyes. Next, the sets of photographs of the participant's own body, of the other female's body, and the scrambled images, which had been presented to the participants previously in the scanner, were again shown to the participants on a laptop computer. Immediately after the presentation of each of the three sets of photographs, the participants were asked to answer the Positive and Negative Affect Schedule in order to assess the emotions that they had experienced when viewing the photographs. The study protocol was approved by the local ethics committee.

\section{Statistical Analyses}

Brain-imaging data were analyzed using SPM 8. Analysis began with slice time correction, followed by motion correction. Each of the scans was realigned to the first scan of the session. All acquired images were normalized to the standard brain of the Montreal Neurological Institute (MNI) as provided by SPM 8. Afterward, images were smoothed using a Gaussian kernel of $8 \mathrm{~mm}$. A general linear model was applied to these data. Contrasts of target stimuli, with the fixation condition serving as an implicit baseline, were calculated in the first-level analysis. First-level contrast images of bodies against chairs (stimulus materials as provided by Downing et al., 2001) were fed into a second-level analysis using a one-sample $t$ test in order to localize the two body-selective brain areas. All the analyses treated subjects as a random effect. Since the signal changes in the right and the left EBA and FBA were considered, the ROI-based analyses are a series of four analyses. The percentages of signal changes to the three stimulus conditions were extracted using the Marsbar software package (marsbar.sourceforge .net/) for each of the three conditions. Using the SPSS 17 software package, signal changes were compared using a three-way ANOVA with the factors of stimulus (own body, other female's body, and scrambled images), region (EBA and FBA), and side (right and left hemisphere). In order to follow up the three-way interactions, an additional two-way ANOVA was calculated separately for the EBA and the FBA. Post hoc one-sample $t$ tests (repeated measures) were calculated separately for each hemisphere in order to further specify the results. In a further analysis, contrast images of looking at one's own body and of looking at the other female's body were fed into second-level one-sample $t$ tests in order to look for identity effects. In a first step, a mask was used that allowed us to focus on the right and the left EBA and FBA on the basis of the localizer analysis mentioned above. Within the mask, data were thresholded at $p<.001$ (uncorrected), with an extent threshold of 8 voxels. In a second step, a whole-brain analysis was performed using a familywise error (FWE) correction. In this analysis, data were thresholded at $p<.05$, with an extent threshold of 8 voxels. The resulting coordinates were transformed into the space defined by Talairach and Tournoux (1988), using the algorithm suggested by Brett (http:// imaging.mrc-cbu.cam.ac.uk/downloads/MNI2tal/mni2tal.m). Differences between the conditions in the scores on the Positive and Negative Affect Schedule were analyzed using one-sample $t$ tests (repeated measures). For the two scales of the Positive and Negative Affect Schedule, we correlated (Pearson coefficient) the difference between the conditions of looking at one's own body and the other female's body with the difference in the signal changes between these two conditions in the EBA and in the FBA.

\section{RESULTS}

\section{Sample Characteristics}

The mean age of the $N=31$ females was $M=27.06$ years $(S D=7.94)$. The sample showed a mean body height of $M=168.81 \mathrm{~cm}(S D=0.06)$ and a mean body mass index of $M=20.51 \mathrm{~kg} / \mathrm{m}^{2}(S D=4.36)$.

\section{Localization of the EBA and FBA}

When the brain responses to looking at the standard body pictures used as target stimuli and the chairs used as control stimuli (Downing et al., 2001) were compared in order to localize the two body-selective brain areas 
Table 1

Comparison of Activation Maps in Response to Viewing Pictures of Various

Human Bodies and Chairs (Downing, Jiang, Shuman, \& Kanwisher, 2001)

As Localizer Stimuli in Order to Determine the Extrastriate Body Area and the Fusiform Body Area, Using a One-Sample $t$ Test

(Random Effects Analysis; $\boldsymbol{p}<.001$, Uncorrected)

\begin{tabular}{|c|c|c|c|c|c|c|}
\hline \multirow[b]{2}{*}{ Structure } & \multirow{2}{*}{$\begin{array}{c}\text { Probable } \\
\text { Brodmann } \\
\text { Area }\end{array}$} & \multicolumn{3}{|c|}{ MNI Coordinates } & \multirow[b]{2}{*}{$k$} & \multirow[b]{2}{*}{$t$ Value } \\
\hline & & $x$ & $y$ & $z$ & & \\
\hline \multicolumn{7}{|l|}{ Bodies $>$ chairs } \\
\hline Middle occipital gyrus & 19 & 48 & -74 & 4 & 1,151 & 6.96 \\
\hline Inferior temporal gyrus & - & 48 & -72 & -4 & & 6.35 \\
\hline Middle temporal gyrus & 39 & 44 & -66 & 18 & & 5.20 \\
\hline Fusiform gyrus & 37 & 40 & -48 & -22 & 162 & 5.83 \\
\hline Middle temporal gyrus & 37 & -42 & -64 & 6 & 385 & 5.27 \\
\hline Superior temporal gyrus & 22 & -48 & -60 & 16 & & 4.49 \\
\hline Middle temporal gyrus & 39 & -52 & -68 & 12 & & 4.48 \\
\hline Anterior cingulate & 25 & 0 & 14 & -4 & 12 & 4.79 \\
\hline Postcentral gyrus & 5 & 44 & -44 & 64 & 19 & 4.76 \\
\hline Inferior temporal gyrus & 37 & -40 & -66 & -10 & 18 & 4.59 \\
\hline Precentral gyrus & 6 & 38 & -4 & 32 & 29 & 4.39 \\
\hline Precentral gyrus & 6 & 32 & 2 & 32 & & 4.15 \\
\hline
\end{tabular}

Note-MNI coordinates, coordinates according to the reference brain of the Montreal Neurological Institute provided by SPM $8 ; k$, number of voxels in a cluster.

EBA and FBA, higher activations were found in the right middle occipital gyrus (BA 19), the bilateral inferior temporal gyrus (BA 37), the bilateral middle temporal gyrus (BAs 37, 39), the right fusiform gyrus (BA 37), the left superior temporal gyrus (BA 22), the medial anterior cingulate (BA 25), the right postcentral gyrus (BA 5), and the right precentral gyrus (BA 6). Signal changes from the whole cluster were extracted for the following coordinates: $x=42, y=-64, z=6$ for the right EBA; $x=-42$, $y=-64, z=6$ for the left EBA; $x=40, y=-48, z=$ -22 for the right FBA; and $x=-40, y=-48, z=-22$ for the left FBA (MNI coordinates; see Table 1).

\section{Percentages of Signal Changes in the EBA and FBA}

The three-way ANOVA with the signal changes located by the standard stimuli (Downing et al., 2001) as dependent variables revealed a significant three-way stimulus $\times$ region $\times$ side interaction $[F(2,58)=3.79$, $p=.028]$, as well as significant two-way stimulus $\times$ side $[F(2,58)=6.78, p=.002]$ and region $\times$ side $[F(1,29)=$ $11.44, p=.002]$ interactions. The stimulus $\times$ region interaction $[F(2,58)=1.66, p=.199]$ was not significant. The main effects of stimulus $[F(2,58)=75.87, p<.001]$, region $[F(1,29)=27.20, p<.001]$, and side $[F(1,29)=$ $17.75, p<.001]$ were significant. To follow up the threeway interaction, additional tests were conducted for the EBA and the FBA separately.

For the EBA, the two-way ANOVA revealed a significant stimulus $\times$ side interaction $[F(2,29)=33.18, p<.001]$, as well as significant main effects of stimulus $[F(2,29)=$ $90.07, p<.001]$ and side $[F(1,30)=96.85, p<.001]$. The post hoc $t$ tests revealed stronger signal changes induced by viewing one's own body $[M=0.1296, S D=$ $0.0441 ; t(30)=15.00, p<.001]$ and the other female's body $[M=0.1162, S D=0.0390 ; t(30)=10.56, p<$ $.001]$, as compared with viewing the scrambled images
$(M=0.0138, S D=0.0323)$. The direct contrast between the condition of looking at one's own body and the other female's body indicated a stronger activation of the right EBA when one's own body was viewed $[t(30)=2.14$, $p=.041]$. For the left EBA, the post hoc $t$ test revealed a higher activation in the conditions of looking at one's own body $[M=0.0592, S D=0.0335 ; t(30)=7.96, p<.001]$ and at the other female's body $[M=0.0537, S D=0.0323$; $t(30)=5.88, p<.001]$, as compared with viewing the scrambled images $(M=0.0005, S D=0.0311)$. The contrast in EBA activation in the left hemisphere between looking at one's own body and the other female's body revealed no statistically significant difference $[t(30)=$ $1.11, p=.275$; see Figure 2].

In the two-way ANOVA for the FBA, the stimulus $\times$ side interaction $[F(2,28)=0.14, p=.871]$ and the main effect of side $[F(1,29)=0.54, p=.469]$ did not reach the sig-

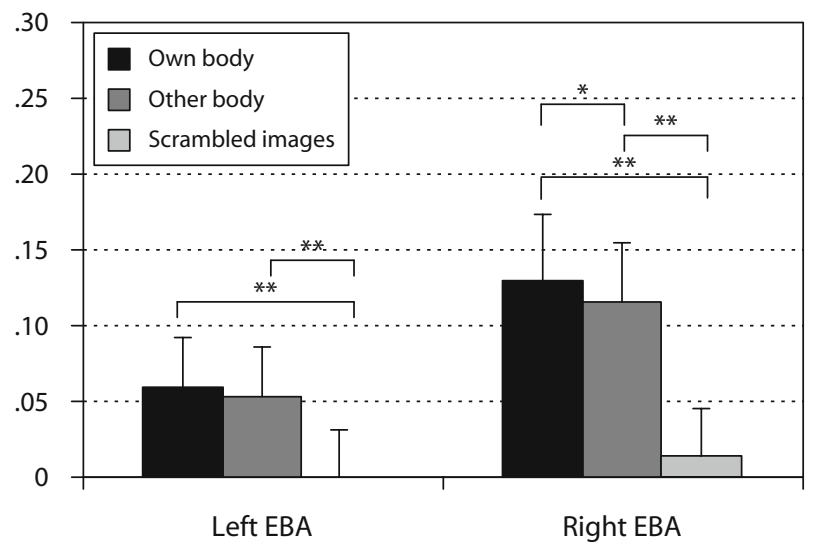

Figure 2. Percentages of signal changes in the right and left extrastriate body area (EBA) in the three stimulus conditions (MNI: right hemisphere, $x=42, y=-64, z=6$; left hemisphere, $x=$ $-42, y=-64, z=6$ ). ${ }^{*} p<.05$. ${ }^{* *} p<.001$. 


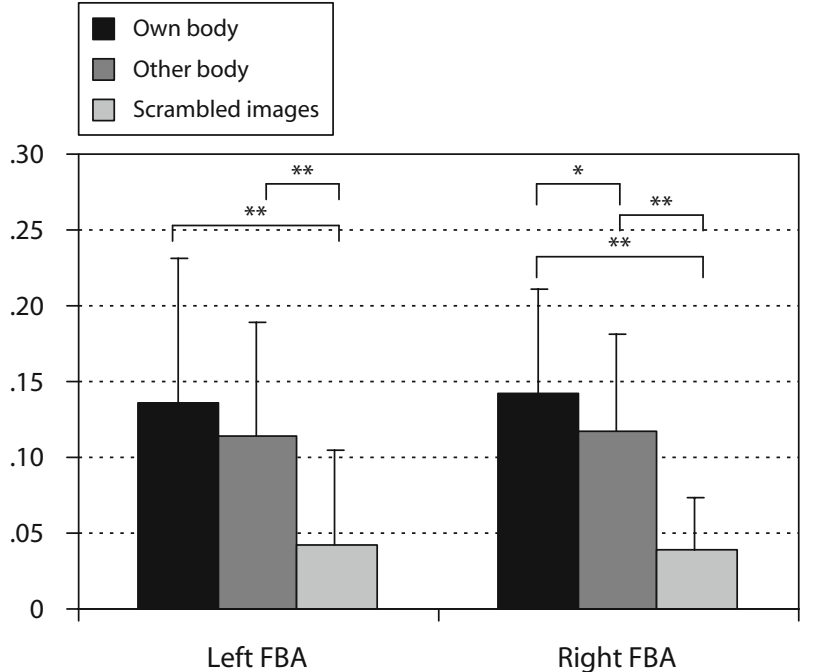

Figure 3. Percentages of signal changes in the right and left fusiform body area (FBA) in the three stimulus conditions (MNI: right hemisphere, $x=40, y=-48, z=-22$; left hemisphere, $x=$ $-40, y=-48, z=-22) . \quad{ }^{*} p<.05 .{ }^{* *} p<.001$.

nificance level, but the main effect of stimulus $[F(2,28)=$ $53.31, p<.001]$ was significant. Post hoc $t$ tests for the right FBA revealed a more pronounced activation on the right side in the conditions of looking at one's own body $[M=0.1419, S D=0.0689 ; t(29)=9.03, p<.001]$ and at the other female's body $[M=0.1169, S D=0.0646$; $t(29)=6.12, p<.001]$, as compared with viewing the scrambled images $(M=0.0392, S D=0.0350)$. The contrast between the condition of viewing one's own body and the other female's body indicated a stronger activation of the right FBA for looking at one's own body $[t(29)=$ $2.80, p=.009]$. Post hoc $t$ tests for the left FBA revealed stronger signal changes induced by viewing one's own body $[M=0.1364, S D=0.0943 ; t(30)=6.69, p<.001]$ and the other female's body $[M=0.1135, S D=0.0761$; $t(30)=4.03, p<.001]$, as compared with viewing the scrambled images $(M=0.0424, S D=0.0624)$. The direct comparison between the activation of the FBA in the left hemisphere between looking at one's own body and the other female's body was not significant $[t(30)=1.45, p=$ .158; see Figure 3].

\section{Whole-Brain Analysis}

Own body $>$ other body. Within the mask, we found stronger activations in the right EBA $(x=54, y=-58$, $z=-4 ; k$ [number of voxels in cluster] $=39 ; t=3.89$ ) and in the right FBA $(x=36, y=-46, z=-20 ; k=100$; $t=3.77$ ) for looking at one's own body, as compared with viewing the other female's body. In the FWE-corrected whole-brain analysis contrasting brain activations in the condition of looking at pictures of one's own body with those in the condition of viewing the images of the other female, stronger activations were found in the right anterior cingulate $(x=6, y=28, z=22 ; k=93 ; t=6.68)$ and in the right claustrum extending to the insula $(x=34$, $y=-2, z=14 ; k=45 ; t=6.64$; see Figure 4).
Other body $>$ own body. No brain region was found to be activated more strongly for looking at the pictures of the other female's body, as compared with viewing one's own body, either in the hypothesis-driven ROI analysis using the bilateral mask for the EBA and FBA or in the FWE-corrected whole-brain analysis.

\section{Positive and Negative Affect Schedule}

Positive affect. As indicated by the data of the Positive and Negative Affect Schedule, higher positive affect scores were observed for looking at one's own body $[M=$ $24.39, S D=6.21 ; t(30)=6.23, p<.001]$ and at the other female's body $[M=22.45, S D=6.00 ; t(30)=4.90, p<$ $.001]$, as compared with viewing the scrambled images $(M=16.77, S D=6.52)$. When we directly compared the conditions of viewing one's own body, as compared with the other female's body, a higher degree of positive affect was found for looking at one's own body $[t(30)=2.39$, $p=.024]$.

Negative affect. Significantly higher scores in the condition of looking at one's own body $(M=15.00, S D=$ 5.67), as compared with viewing the scrambled images $[M=10.90, S D=1.42 ; t(30)=4.11, p<.001]$, were found for negative affect. No differences were observed between the conditions of looking at the other female's body $(M=12.03, S D=4.17)$ and viewing the scrambled image $[t(30)=1.58, p=.125]$. When viewing one's own body, as compared with viewing the other female's body, the participants displayed significantly higher negative affect scores $[t(30)=4.30, p<.001]$.

\section{Correlational Analyses}

The difference between the affective responses to looking at one's own body and at the other female's body did not significantly correlate with the activation difference between viewing one's own body and the other female's body, on the basis of the extracted signal changes in the

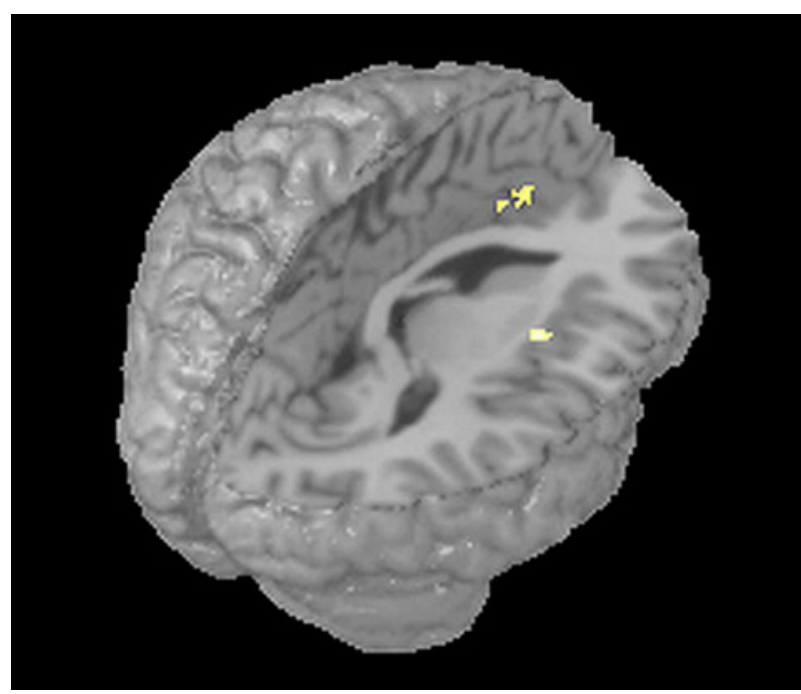

Figure 4. Activation differences depicting the own body $>$ other body contrast in a whole-brain analysis. 
right EBA (positive affect, $r=.057, p=.761$; negative affect, $r=.088, p=.637$ ), the left EBA (positive affect, $r=-.010, p=.956$; negative affect, $r=.229, p=.216$ ), the right FBA (positive affect, $r=-.142, p=.455$; negative affect, $r=.000, p=.999$ ), and the left FBA (positive affect, $r=-.033, p=.859$; negative affect, $r=.138$, $p=.459)$.

\section{DISCUSSION}

The aim of the present study was to shed light on differences in activation in the EBA and the FBA elicited by visual processing of one's own body and another female's body in healthy females. Since the EBA and FBA are functionally, rather than anatomically, defined brain areas, we located the bilateral EBA and FBA using the stimulus materials provided by Downing et al. (2001) in order to extract the signal changes in the various conditions and to compare them. In these analyses, identitydependent differences, with a higher neuronal response for looking at one's own body than for viewing the other female's body, emerged in the right EBA and FBA. This finding was confirmed by the hypothesis-driven ROI analysis using the bilateral mask covering the EBA and FBA. These results, suggesting that the right EBA response differs depending on whether one is viewing oneself or another person, stand in contrast to the findings of previous research demonstrating no differences in the EBA activation between looking at one's own body and another person's body (Chan et al., 2004; Hodzic et al., 2009), which might be attributable to methodological aspects. Beyond differences in stimulus material (e.g., photographs taken with participants dressed in jeans and a T-shirt in the study by Chan et al. [2004] vs. in a bikini in the present study) and in statistical approaches, the size and composition of the samples might explain the conflicting results, since the sample examined in the present study was larger and, due to the restriction to females, more homogeneous. In view of this, and because the differences in EBA and FBA activation between the participants' looking at the pictures of their own body, as compared with those of the other female's body, were quantitatively rather small in relation to the EBA and FBA responses in general, a relatively high statistical power seems to be necessary to detect these apparently moderate differences. Our results are in accordance with those of Myers and Sowden (2008), who reported an enhanced EBA activation on the right side to viewing oneself; however, in contrast to the present study, they used body parts and not whole bodies as stimulus materials. The findings of the present study regarding the laterality of the responses of the two body-selective brain areas to viewing oneself can be regarded in the context of the results in Chan et al. and Saxe et al. (2006), insofar as these previous studies consistently reported an enhanced activation of the EBA in the right hemisphere, but not in the left hemisphere, from the egocentric, as compared with the allocentric, perspective. Chan et al. suggested that the preference in the right hemisphere for allocentric views may indicate an early stage in the social visual system.
These results beg the question of which mechanisms this self-other difference in the activity of the right EBA and FBA is based on. Since a previous study by Peelen, Atkinson, Andersson, and Vuilleumier (2007) showed that emotional body stimuli with positive or negative valence activate the EBA and FBA more strongly than do neutral body stimuli, it can be hypothesized that the present differences between the visual processing of one's own body and the other female's body might be influenced by emotional states. This suggestion is probably reflected by the results of the whole-brain analysis that revealed that in the condition of viewing oneself, as compared with looking at the other female, stronger activations in the right anterior cingulate and in the right claustrum extending to the insula could be found. These and vicinal brain areas have been shown in previous research to be involved not only in selfrelated tasks (e.g., Farrer \& Frith, 2002; Lamm, Batson, \& Decety, 2007; Modinos, Ormel, \& Aleman, 2009; Ochsner et al., 2004), but also in emotion processing (e.g., Allman, Hakeem, Erwin, Nimchinsky, \& Hof, 2001; Bush, Luu, \& Posner, 2000; Craig, 2004; Sehlmeyer et al., 2009). Furthermore, data gained from the Positive and Negative Affect Schedule indicated a higher emotional involvement with positive as well as with negative valence for looking at one's own body, as compared with viewing the other female's body. However, we did not find a significant correlation of the activation difference to viewing one's own body and the other female's body in the EBA and FBA with the affective response to looking at one's own body and the other female's body.

On the basis of previous findings indicating a modulation of the activity of the extrastriate cortex by attentional processes (Corbetta, Miezin, Dobmeyer, Shulman, \& Petersen, 1990; Moran \& Desimone, 1985), the self-other difference in EBA and FBA activity might be driven by the extent of attention directed toward the pictures. However, since we found significant differences between viewing oneself and the other female for the two body-selective brain areas exclusively on the right side, and general factors such as attention are assumed to influence the activity in both hemispheres (Chan et al., 2004), it seems improbable that the self-other differences in the EBA and FBA are mainly related to attention.

A third mechanism possibly underlying the self-other discrepancy in the body-selective brain areas might be motor resonance, since it was found in previous research that the EBA activity responds to self-produced movements of the body, even in the absence of visual feedback from the movement (Astafiev, Stanley, Shulman, \& Corbetta, 2004). Because these findings imply that the EBA receives signals not only from the retina, but also from brain regions involved in the generation of movements, Jeannerod (2004) inferred that the EBA can distinguish between movements generated by oneself and by another person. Since exclusively static pictures of bodies were used in the present study, future research on dynamic aspects should further clarify this issue.

Contrary to our hypothesis, in the additional wholebrain analysis, we did not find significant differences in 
the middle and inferior frontal gyrus, as well as the precuneus, when comparing the activations elicited by looking at oneself and by the other female. This result might be due to the relatively conservative correction applied to these contrasts.

When the data are interpreted, it has to be considered that for homogeneity reasons, we restricted our sample to females. Therefore, it is unclear to what extent the results can be generalized to males, especially given the fact that previous research has demonstrated gender differences in the processing of word and image stimuli of bodies, with females displaying a higher limbic involvement, relative to men (Kurosaki, Shirao, Yamashita, Okamoto, \& Yamawaki, 2006; Shirao, Okamoto, Mantani, Okamoto, \& Yamawaki, 2005).

To conclude, the results of the present study demonstrated that in the right hemisphere, the EBA and FBA seem to have the property of visual self-other discrimination. Therefore, the findings suggest that these two bodyselective brain areas play a role in the assignment of body identity and social cognition.

\section{AUTHOR NOTE}

None of the authors reports any conflict of interest regarding the research reported in this article. The authors thank Claudia Moswald, Annika Vogt, Karin Liebenrodt, Serban Mateiescu, Marie Hennecke, Lena Schloßmacher, and Kathrin Hubbert for helpful assistance during image acquisition and data management. Correspondence concerning this article should be addressed to S. Vocks, Department of Psychology, Clinical Psychology and Psychotherapy, Ruhr University Bochum, Universitätsstrasse 150, D-44780 Bochum, Germany (e-mail: silja.vocks@ ruhr-uni-bochum.de).

\section{REFERENCES}

Allman, J. M., Hakeem, A., Erwin, J. M., Nimchinsky, E., \& Hof, P. (2001). The anterior cingulate cortex: The evolution of an interface between emotion and cognition. In A. R. Damasio, A. Harrington, J. Kagan, B. S. McEwen, H. Moss, \& R. Shaikh (Eds.), Unity of knowledge: The convergence of natural and human science (Annals of the New York Academy of Sciences, Vol. 935, pp. 107-117). New York: New York Academy of Sciences.

Astafiev, S. V., Stanley, C. M., Shulman, G. L., \& Corbetta, M. (2004). Extrastriate body area in human occipital cortex responds to the performance of motor actions. Nature Neuroscience, 7, 542-548.

Bush, G., LuU, P., \& Posner, M. (2000). Cognitive and emotional influences in anterior cingulate cortex. Trends in Cognitive Sciences, 4, 215-222.

Chan, A. W. Y., Peelen, M. V., \& Downing, P. E. (2004). The effect of viewpoint on body representation in the extrastriate body area. NeuroReport, 15, 2407-2410.

Corbetta, M., Miezin, F., Dobmeyer, S., Shulman, G., \& PeTERSEN, S. (1990). Attentional modulation of neural processing of shape, color, and velocity in humans. Science, 248, 1556-1559.

Craig, A. D. (2004). Human feelings: Why are some more aware than others? Trends in Cognitive Sciences, 8, 239-241.

Downing, P. E., Chan, A. W.-Y., Peelen, M. V., Dodds, C. M., \& KANwisher, N. (2006). Domain specificity in visual cortex. Cerebral Cortex, 16, 1453-1461.

Downing, P. E., Jiang, Y., Shuman, M., \& Kanwisher, N. (2001). A cortical area selective for visual processing of the human body. Science, 293, 2470-2473.

Dricot, L., Sorger, B., Schiltz, C., Goebel, R., \& Rossion, B. (2008). Evidence for individual face discrimination in non-face selective areas of the visual cortex in acquired prosopagnosia. Behavioural Neurology, 19, 75-79.
Fairburn, C. G., \& Cooper, G. T. (1993). The Eating Disorder Examination. In C. F. Fairburn \& G. T. Wilson (Eds.), Binge eating: Nature, assessment and treatment (pp. 317-332). New York: Guilford.

FARRER, C., \& Frith, C. D. (2002). Experiencing oneself vs another person as being the cause of an action: The neural correlates of the experience of agency. NeuroImage, 15, 596-603.

Gillihan, S. J., \& FARAH, M. J. (2005). Is self special? A critical review of evidence from experimental psychology and cognitive neuroscience. Psychological Bulletin, 131, 76-97.

Hilbert, A., Tuschen-Caffier, B., Karwautz, A., Niederhofer, N., \& Munsch, S. (2007). Eating Disorder Examination Questionnaire: Evaluation der deutschsprachigen Übersetzung [Eating Disorder Examination Questionnaire: Evaluation of the German translation]. Diagnostica, 53, 144-154.

Hodzic, A., Muckli, L., Singer, W., \& Stirn, A. (2009). Cortical responses to self and others. Human Brain Mapping, 30, 951-962.

JEANNERoD, M. (2004). Visual and action cues contribute to the selfother distinction. Nature Neuroscience, 7, 422-423.

Kanwisher, N., McDermott, J., \& Chun, M. M. (1997). The fusiform face area: A module in human extrastriate cortex specialized for face perception. Journal of Neuroscience, 17, 4302-4311.

Krohne, H. W., Egloff, B., Kohlmann, C.-W., \& Tausch, A. (1996). Untersuchungen mit einer deutschen Version der "Positive and Negative Affect Schedule" (PANAS) [Studies with a German version of the "Positive and Negative Affect Schedule" (PANAS)]. Diagnostica, 42, 139-156.

Kurosaki, M., Shirao, N., Yamashita, H., Okamoto, Y., \& YamaWAKI, S. (2006). Distorted images of one's own body activate the prefrontal cortex and limbic/paralimbic system in young women: A functional magnetic resonance imaging study. Biological Psychiatry, 59, 380-386.

Lamm, C., Batson, C. D., \& Decety, J. (2007). The neural substrate of human empathy: Effects of perspective-taking and cognitive appraisal. Journal of Cognitive Neuroscience, 19, 42-58.

Modinos, G., Ormel, J., \& Aleman, A. (2009). Activation of anterior insula during self-reflection. PLoS ONE, 4, e4618.

Moran, J., \& Desimone, R. (1985). Selective attention gates visual processing in the extrastriate cortex. Science, 229, 782-784.

Morris, J. P., Pelphrey, K. A., \& McCarthy, G. (2006). Occipitotemporal activation evoked by the perception of human bodies is modulated by the presence or absence of the face. Neuropsychologia, 44, 1919-1927.

Myers, A., \& Sowden, P. T. (2008). Your hand or mine? The extrastriate body area. NeuroImage, 42, 1669-1677.

Northoff, G., Heinzel, A., De Greck, M., Bermpohl, F., DobroWOLNY, H., \& PANKSEPP, J. (2006). Self-referential processing in our brain: A meta-analysis of imaging studies on the self. NeuroImage, 31, 440-457.

Northoff, G., Schneider, F., Rotte, M., Matthiae, C., TempelMANN, C., WiEbKing, C., ET AL. (2009). Differential parametric modulation of self-relatedness and emotions in different brain regions. Human Brain Mapping, 30, 369-382.

Ochsner, K. N., Knierim, K., Ludlow, D. H., Hanelin, J., Ramachandran, T., Glover, G., \& Mackey, S. C. (2004). Reflecting upon feelings: An fMRI study of neural systems supporting the attribution of emotion to self and other. Journal of Cognitive Neuroscience, 16, 1746-1772.

OLDFIELD, R. C. (1971). The assessment and analysis of handedness: The Edinburgh Inventory. Neuropsychologia, 9, 97-113.

Peelen, M. V., Atkinson, A. P., Andersson, F., \& Vuilleumier, P. (2007). Emotional modulation of body-selective visual areas. Social Cognitive \& Affective Neuroscience, 2, 274-283.

Peelen, M. V., \& Downing, P. E. (2007). The neural basis of visual body perception. Nature Reviews Neuroscience, 8, 636-648.

Platek, S. M., Wathne, K., Tierney, N. G., \& Thomson, J. W. (2008). Neural correlates of self-face recognition: An effect-location metaanalysis. Brain Research, 1232, 173-184.

Puce, A., Allison, T., Asgari, M., Gore, J. C., \& McCarthy, G. (1996). Differential sensitivity of human visual cortex to faces, letterstrings, and textures: A functional magnetic resonance imaging study. Journal of Neuroscience, 16, 5205-5215. 
SAXe, R., Jamal, N., \& Powell, L. (2006). My body or yours? The effect of visual perspective on cortical body representations. Cerebral Cortex, 16, 178-182.

Sehlmeyer, C., Schöning, S., Zwitserlood, P., Pfleiderer, B., Kircher, T., Arolt, V., \& Konrad, C. (2009). Human fear conditioning and extinction in neuroimaging: A systematic review. PLoS ONE, 4, e5865.

Shirao, N., OKamoto, Y., Mantani, T., Okamoto, Y., \& Yamawaki, S. (2005). Gender differences in brain activity generated by unpleasant word stimuli concerning body image: An fMRI study. British Journal of Psychiatry, 186, 48-53.

Suchan, B., Busch, M., Schulte, D., Grönemeyer, D., Herpertz, S., \& VocKs, S. (2010). Reduction of gray matter density in the extrastriate body area in women with anorexia nervosa. Behavioural Brain Research, 206, 63-67.

Sugiura, M., Sassa, Y., Jeong, H., Miura, N., Akitsuki, Y., Horie, K.,
ET AL. (2006). Multiple brain networks for visual self-recognition with different sensitivity for motion and body part. NeuroImage, $\mathbf{3 2}$, 1905-1917.

TAlairach, J., \& Tournoux, J. (1988). Co-planar stereotaxic atlas of the human brain: 3-dimensional proportional system-An approach to cerebral imaging. New York: Thieme.

Uher, R., Murphy, T., Friederich, H.-C., Dalgleish, T., Brammer, M. J., Giampietro, V., ET AL. (2005). Functional neuroanatomy of body shape perception in healthy and eating-disordered women. Biological Psychiatry, 58, 990-997.

Watson, D., Clark, L. A., \& Tellegen, A. (1988). Development and validation of brief measures of positive and negative affect: The PANAS scales. Journal of Personality \& Social Psychology, 54, 1063-1070.

(Manuscript received April 7, 2009;

revision accepted for publication April 9, 2010.) 